\title{
Antisense Knockdown of the Glial Glutamate Transporter GLT-1, But Not the Neuronal Glutamate Transporter EAAC1, Exacerbates Transient Focal Cerebral Ischemia-Induced Neuronal Damage in Rat Brain
}

\author{
Vemuganti L. Raghavendra Rao, ${ }^{1,2}$ Aclan Dogan, ${ }^{1}$ Kathryn G. Todd, ${ }^{4}$ Kellie K. Bowen, ${ }^{1}$ Bum-Tae Kim, ${ }^{1,5}$ \\ Jeffrey D. Rothstein, ${ }^{6}$ and Robert J. Dempsey ${ }^{1,3}$ \\ ${ }^{1}$ Department of Neurological Surgery and ${ }^{2}$ Cardiovascular Research Center, University of Wisconsin-Madison, Madison, \\ Wisconsin 53792, 3William S. Middleton Memorial Veterans Administration Hospital, Madison, Wisconsin 53792, \\ ${ }^{4}$ Department of Psychiatry, University of Alberta, Edmonton, Canada T6G 2B7, ${ }^{5}$ Department of Neurosurgery, \\ Soonchunhyang University Hospital, Seoul, Korea, and ${ }^{6}$ Department of Neurology, The Johns Hopkins University School \\ of Medicine, Baltimore, Maryland 21287
}

Transient focal cerebral ischemia leads to extensive neuronal damage in cerebral cortex and striatum. Normal functioning of glutamate transporters clears the synaptically released glutamate to prevent excitotoxic neuronal death. This study evaluated the functional role of the glial (GLT-1) and neuronal (EAAC1) glutamate transporters in mediating ischemic neuronal damage after transient middle cerebral artery occlusion (MCAO). Transient MCAO in rats infused with GLT-1 antisense oligodeoxynucleotides (ODNs) led to increased infarct volume (45 $\pm 8 \% ; p<0.05)$, worsened neurological status, and in- creased mortality rate, compared with GLT-1 sense/random ODN-infused controls. Transient MCAO in rats infused with EAAC1 antisense ODNs had no significant effect on any of these parameters. This study suggests that GLT-1, but not EAAC1, knockdown exacerbates the neuronal death and thus neurological deficit after stroke.

Key words: antisense knockdown; EAAC1; focal cerebral ischemia; GLT-1; glutamate transporters; middle cerebral artery occlusion; neuronal damage; stroke
After transient focal cerebral ischemia, the progression of infarct evolution and neuronal damage depends on the duration of ischemia and reperfusion (Lipton, 1999). The size of the infarct increases up to $24 \mathrm{hr}$ of reperfusion after a $2 \mathrm{hr}$ transient middle cerebral artery occlusion (MCAO) in rats (Zhang et al., 1994). Immediately after transient MCAO, the extracellular glutamate concentrations rapidly increase and return to baseline within $1 \mathrm{hr}$ (Takagi et al., 1994; Uchiyama-Tsuyuki et al., 1994). This early release of glutamate is critical to ischemic neuronal damage (Kato and Kogure, 1999; Lipton, 1999).

Uptake into glia and neurons, mediated by a family of highaffinity transporters (GLAST/EAAT1, GLT-1/EAAT2, EAAC1/ EAAT3, EAAT4, and EAAT5), is the only mechanism by which extracellular glutamate is inactivated (Kanai et al., 1997). GLAST and GLT-1 are glial, whereas EAAC1 and EAAT4 are neuronal (Rothstein et al., 1994; Chaudhry et al., 1995). GLT-1 mediates the bulk of the glutamate uptake in forebrain (Robinson, 1999). Protein knockdown studies have shown that GLT-1 and GLAST, but not EAAC1, are essential for maintaining the

\footnotetext{
Received July 12, 2000; revised Dec. 29, 2000; accepted Jan. 2, 2001.

This work was supported by American Heart Association National Grant 9950086N, American Heart Association Wisconsin Affiliate Grant 9806376X, the University of Wisconsin (UW)-Madison Medical School Research Committee Grant 161-9587, and the Department of Neurological Surgery UW-Madison Start-up Grant to V.L.R.R., National Institutes of Health Grants NS28000 and NS31220 to R.J.D., and a Korean Research Foundation grant to B.-T.K. We thank Dr. Peter Lipton (University of Wisconsin-Madison) for comments and suggestions.

Correspondence should be addressed to Dr. Vemuganti L. R. Rao, Department of Neurological Surgery, University of Wisconsin-Madison, F4/309 CSC, 600 Highland Avenue, Madison, WI 53792. E-mail: Vemugant@neurosurg.wisc.edu.

Copyright (C) 2001 Society for Neuroscience 0270-6474/01/211876-08\$15.00/0
}

extracellular glutamate below neurotoxic levels in the normal rat brain (Rothstein et al., 1996).

In addition to clearing the released glutamate, subtypes of glutamate transporters were also proposed to release intracellular glutamate during ischemic conditions (Phillis and O'Regan, 1996). Seki et al. (1999) showed that cell swelling-induced release and reversal of astrocytic glutamate transport contribute to the increased extracellular glutamate in rat striatum after transient forebrain ischemia. Phillis et al. (2000) observed a 50\% reduction in forebrain ischemia-evoked glutamate release by the application of DL-threo- $\beta$-benzyloxyaspartate (DL-TBOA), which is a potent, competitive, nontransported blocker of high-affinity, sodiumdependent glutamate transporters. Reversal of neuronal glutamate transporters, probably localized in glutamatergic presynaptic terminals, was thought to be responsible for glutamate release from hippocampal slices subjected to in vitro ischemia (Rossi et al., 2000). Roettger and Lipton (1996) failed to observe any effect of dihyrokainate (DHK), a nontransportable blocker of the reversed uptake by GLT-1, on ischemia-induced glutamate release from hippocampal slices. These studies suggest that GLT-1 reversal may not be responsible for increased extracellular glutamate concentrations under in vitro ischemic conditions.

Although dysfunctional glutamate reuptake has been proposed to promote the neuronal death after global cerebral ischemia (Torp et al., 1995; Rao et al., 2000) and hypoxic ischemia (Martin et al., 1997; Inage et al., 1998), no studies have examined the functional significance of glutamate transporter subtypes in precipitating the neuronal death after focal cerebral ischemia. This study focused on the effect of antisense knockdown of GLT-1 and 
EAAC1 on the infarct volume, neuronal death, and neurological deficit in spontaneously hypertensive (SHR) rats subjected to transient MCAO. Antisense knockdown of GLT-1, but not EAAC1, exacerbated the ischemic infarct volume and neuronal damage in cerebral cortex and striatum.

\section{MATERIALS AND METHODS}

Adult, male, SHR rats (250-300 gm; Charles River, Wilmington, MA) were used in these studies. Rats were housed and cared for in accordance with the Guide for the Care and Use of Laboratory Animals, U.S. Department of Health and Human Services Publication number 86-23 (revised 1986). All surgical procedures were approved by the Research Animal Resources and Care Committee of the University of Wisconsin-Madison.

\section{Intracerebroventricular administration of oligodeoxynucleotides}

The antisense oligodeoxynucleotides (ODNs) were designed on the basis of the known sequences of rat GLT-1 and EAAC1 (Kanai and Hediger, 1992; Pines et al., 1992) and successfully used previously to specifically knock down the GLT-1 and EAAC1 proteins in rat brain (Rothstein et al., 1996). Sense and random ODNs in which the proportion of each nucleotide is identical to that of the antisense ODN were used as controls. The sequences of the ODNs are 5'-ATC AAC CGA GGG TGC CAA CAA TAT-3' (GLT-1 sense), 5'-ATA TTG TTG GCA CCC TCG GTT GAT-3' (GLT-1 antisense), 5'-AAT TGT GTT AGC CCC CTC TGT TGA-3' (GLT-1 random), 5'-GCT CGG GAT GCG ACT GGC-3' (EAAC1 sense), 5'-GCC AGT CGC ATC CCG AGC-3' (EAAC1 antisense), and 5'-GCG GAT CCG TAC GCC CAG-3' (EAAC1 random). These ODNs were synthesized with a phosphorothioate backbone and purified to analytical grade on HPLC by Oligos Etc. (Wilsonville, OR). The ODNs were infused as described previously (Rothstein et al., 1996). The lyophilized ODNs were reconstituted $(2.5 \mathrm{mg} / \mathrm{ml})$ in artificial CSF (aCSF) containing (in mM) $119 \mathrm{NaCl}, 3.1 \mathrm{KCl}, 1.2 \mathrm{CaCl}_{2}, 1 \mathrm{MgSO}_{4}, 0.50$ $\mathrm{KH}_{2} \mathrm{PO}_{4}, 25 \mathrm{NaHCO}_{3}, 5$ D-glucose, 2.2 urea, $\mathrm{pH}$ 7.4; dialyzed (Spectra/ Por cellulose ester; molecular weight cutoff 2000; Thomas Scientific) overnight at $4^{\circ} \mathrm{C}$; filtered $(0.22 \mu \mathrm{m})$; and filled into osmotic minipumps. Each pump was connected to a stainless steel cannula by peristaltic tubing and primed overnight at $37^{\circ} \mathrm{C}$. The cannula was implanted into the lateral ventricle [coordinates: lateral, $-1.5 \mathrm{~mm}$; anterior-posterior, -0.8 $\mathrm{mm}$; and dorsal-ventral, $-4.8 \mathrm{~mm}$; on the basis of the rat brain atlas of Paxinos and Watson (1998)] and secured to the skull with dental cement. The pump was placed in the skin fold on the neck of the rat under halothane anesthesia. The osmotic minipumps used were Alzet model 2001 (Alza, Palo Alto, CA), which will pump at a rate of $1 \mu \mathrm{l} / \mathrm{hr}$ for $7 \mathrm{~d}$. Thus, each rat received $60 \mu \mathrm{g}$ of ODN per day $\left(2.5 \mu \mathrm{g} \cdot \mu \mathrm{l}^{-1} \cdot \mathrm{hr}^{-1}\right)$. After $5 \mathrm{~d}$ of ODN inf usion, rats were subjected to either transient MCAO ( $1 \mathrm{hr}$ ) or sham operation. After $1 \mathrm{~d}$ of reperfusion, rats were neurologically evaluated and perfused transcardially with buffered paraformaldehyde. The brains were then removed, post-fixed, and cryoprotected. Proper functioning of the osmotic minipumps was confirmed by weighing the filled pumps before implantation and immediately after the rats were killed. The average total volume of pumping was observed to be $139 \pm 6$ $\mu \mathrm{l}$ in $6 \mathrm{~d}(0.97 \pm 0.04 \mu \mathrm{l} / \mathrm{hr} ; n=91)$. Correct placement of the cannula into the lateral ventricle was confirmed by examining the thionine-stained brain slices. The effect of antisense, sense, and random ODN infusion on the levels of GLT-1 and EAAC1 proteins was evaluated by Western blotting as described previously (Rao et al., 1998). In brief, tissue samples were homogenized in ice-cold $25 \mathrm{~mm}$ Tris- $\mathrm{HCl}$ buffer, $\mathrm{pH}$ 7.4, containing $2 \mathrm{~mm}$ EDTA and protease inhibitors [aprotinin, pepstatin-A, leupeptin, bestatin, 4-(2-aminoethyl) benzenesulfonyl fluoride, and transepoxysuccinyl-L-leucylamido(4-guanidino) butane]. The homogenate was centrifuged $\left(70,000 \times g ; 30 \mathrm{~min}\right.$ at $\left.4^{\circ} \mathrm{C}\right)$, and the pellet (membranes) was resuspended in fresh buffer. Proteins were electrophoresed on polyacrylamide gels, transferred onto nitrocellulose, and probed with polyclonal affinity-purified anti-GLT-1 and anti-EAAC1 antibodies and HRPcoupled goat anti-rabbit IgG (Rothstein et al., 1994). The protein bands detected by antibodies were visualized using the ECL Western blotting kit (Amersham Pharmacia Biotech, Piscataway, NJ). Immunoblot intensities were quantified by densitometric scanning using the NIH Image program. A linear signal ratio for quantitative analysis was determined by running protein concentration curves. For each sample, blots were prepared twice. Before immunodetection, the blots were stained with Ponceau-S to confirm the protein loading and transfer efficiency.

\section{Transient focal cerebral ischemia}

Animal preparation. Rats were anesthetized with halothane (induction, $2 \%$; maintenance, $1.2 \%$ ) in an oxygen/nitrous oxide (50:50) mixture. Animals were ventilated mechanically with a rodent ventilator (model 683; Harvard Apparatus, South Natick, MA) through an endotracheal tube (PE-240 polyethylene tubing). The left femoral artery was cannulated for continuous monitoring of arterial blood pressure and to obtain the measurements of $\mathrm{pH}, \mathrm{Pa}_{\mathrm{O}_{2}}, \mathrm{~Pa}_{\mathrm{CO}_{2}}$, hemoglobin, and blood glucose concentration (i-STAT; Sensor Devices, Waukesha, WI). $\mathrm{Pa}_{\mathrm{O}_{2}}$ and $\mathrm{Pa}_{\mathrm{CO}_{2}}$ were maintained between 100-200 and 30-40 $\mathrm{mm} \mathrm{Hg}$, respectively.

Transient middle cerebral artery occlusion. MCAO was conducted by an intraluminal suture technique as described previously (Longa et al., 1989; Dogan et al., 1999). In brief, the left common carotid artery (CCA), external carotid artery (ECA), and internal carotid artery (ICA) were exposed through a ventral midline incision. A 3-0 monofilament nylon suture with a rounded tip was introduced into the ECA lumen and gently advanced to the ICA until slight resistance was felt and a reduction in regional cerebral blood flow ( $\mathrm{rCBF}$ ) was seen. The $\mathrm{rCBF}$ dropped to $14-19 \%$ of the baseline in $40-50 \mathrm{sec}$ and remained at that level throughout the occlusion period. After $1 \mathrm{hr}$ of occlusion, the suture was withdrawn to restore the CCA-ICA-MCA blood flow [confirmed by laser Doppler flowmeter (Vasamedics, St. Paul, MN)]. In $<5$ min after the withdrawal of the suture, the $\mathrm{rCBF}$ returned to the baseline level and remained unchanged through 90 min of reperfusion. Body and cranial temperatures were maintained with a heating blanket and a lamp at $37-38$ and $36-37^{\circ} \mathrm{C}$, respectively, during the $1 \mathrm{hr}$ of occlusion and $90 \mathrm{~min}$ of reperfusion. After recovering from anesthesia, rats were returned to their cages with ad libitum access to food and water.

$r C B F$ measurement. Changes in $\mathrm{rCBF}$ were recorded as described previously (Dogan et al., 1999). Before the MCAO was conducted, rats were placed in the stereotaxic frame, and a craniectomy $(4 \mathrm{~mm}$ in diameter; 2-4 $\mathrm{mm}$ lateral and 1-2 $\mathrm{mm}$ caudal to bregma) was performed with extreme care over the MCA territory using a trephine. The dura was left intact. A laser Doppler flowmeter probe (model PD-434; Vasamedics) was placed on the surface of the ipsilateral cortex (ischemic area) and fixed to the periosteum with a 4-0 silk suture. The probe was connected to a laser flowmeter device (Laserflo blood perfusion monitor BPM 403A; TSI, St. Paul, MN). To confirm that antisense treatment had not changed the $\mathrm{rCBF}$ during ischemia, end ischemic $\mathrm{rCBF}$ was measured in additional cohorts by 4 -iodo- $\left[N\right.$-methyl- $\left.{ }^{14} \mathrm{C}\right]$ antipyrine $\left(\left[{ }^{14} \mathrm{C}\right] \mathrm{AIP}\right)$ autoradiography as described previously (Alkayed et al., 1998; Rusa et al., 1999). For this, in a separate set of sense- and antisense-inf used rats, the laser Doppler flowmeter probes were attached, arterial and venous femoral catheters were inserted, and the MCA was occluded. At $60 \mathrm{~min}$ of occlusion, arterial blood pressure, $\mathrm{P}_{\mathrm{O}_{2}}, \mathrm{P}_{\mathrm{CO}_{2}}$, and $\mathrm{pH}$ were measured, and $40 \mu \mathrm{Ci}$ of $\left(\left[{ }^{14} \mathrm{C}\right] \mathrm{AIP}\right.$ (specific activity, $54 \mathrm{mCi} / \mathrm{mmol}$; Amersham Pharmacia Biotech, Arlington Heights, IL) in $0.8 \mathrm{ml}$ of isotonic saline were infused intravenously for $45 \mathrm{sec}$. Simultaneously, the arterial catheter was opened, and 15 free-flowing $20 \mu \mathrm{l}$ samples were collected into heparin-coated tubes. With the filament still in place and the laser Doppler indicating the occlusion, the rat was decapitated, and the brain was snap-frozen by dipping in isopentane cooled to $-30^{\circ} \mathrm{C}$ and stored at $-80^{\circ} \mathrm{C}$. Each brain was sectioned $(20 \mu \mathrm{m}$ thick) on a cryostat, and the sections from four coronal levels $(+0.5,-0.9,-2.1$, and -3.9 from bregma) were collected and exposed for 1 week to Hyperfilm $\beta$ max (Amersham Pharmacia Biotech) together with ${ }^{14} \mathrm{C}$ standards. The blood samples were decolorized with tissue solubilizer, and the radioactivity was estimated by liquid scintillation spectrometry. The autoradiographic images were digitized using the MCID image analysis system (Imaging Research, St. Catherines, Ontario, Canada), and the rates of rCBF were determined as previously described (Alkayed et al., 1998).

Histopathology. Each brain was sectioned coronally ( $40 \mu \mathrm{m}$ thick at an interval of $320 \mu \mathrm{m}$ ), stained with thionine, and scanned using the NIH Image program. The volume of the ischemic lesion was computed by the numeric integration of data from 16 to 19 serial sections in respect to the sectional interval. To account for the cerebral edema and differential shrinkage resulting from tissue processing, the injury volumes were corrected using the following formula: corrected injury volume $=$ contralateral hemisphere volume - (ipsilateral hemisphere volume - measured injury volume) (Swanson et al., 1990).

Neurological evaluation. Neurological deficits were evaluated on a six-point scale (Longa et al., 1989) before transient MCAO and at $1 \mathrm{~d}$ of reperfusion (before the animals were killed) by an investigator blinded to the study groups. A score of 0 suggests no neurological deficit (normal), 1 suggests mild neurological deficit (failure to extend right forepaw 


\section{GLT-1 Knockdown}

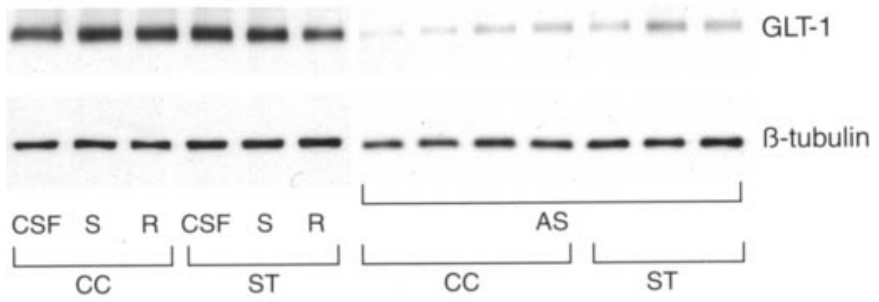

EAAC1 Knockdown

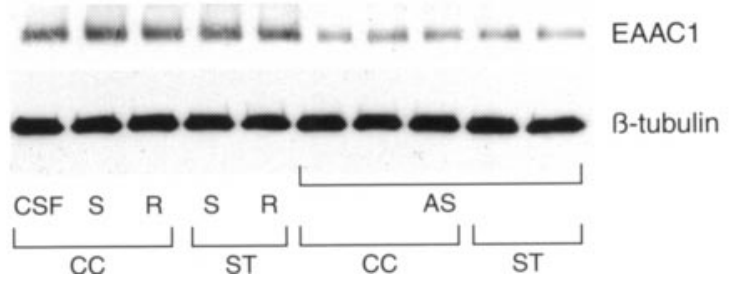

Figure 1. Western blot analysis of GLT-1 and EAAC1 protein levels in rats infused with GLT-1 and EAAC1 antisense, sense, and random ODNs. The amount of protein loaded per lane was $2 \mu \mathrm{g}$ in the GLT-1 gels and $15 \mu \mathrm{g}$ in the EAAC1 gels. Figure shows representative samples from each group. $C C$, Cerebral cortex; $S T$, striatum; $C S F$, artificial CSF; $S$, sense ODN; $R$, random ODN; and $A S$, antisense ODN. GLT-1 antisense infusion resulted in a significant decrease in GLT-1 protein levels compared with GLT-1 sense/random-infused controls. Similarly, EAAC1 antisense infusion significantly decreased EAAC1 protein levels compared with EAAC1 sense/random-infused controls. No significant changes were observed in the $\beta$-tubulin protein levels after the infusion of GLT-1 or EAAC1 sense/random/antisense.

fully), 2 suggests moderate neurological deficit (circling to the right), 3 suggests severe neurological deficit (falling to the right), and 4 suggests very severe neurological deficit (the rat did not walk spontaneously and had a depressed level of consciousness).

Statistical analysis. The ipsilateral value was compared with the contralateral value and the sham-operated value using one-way ANOVA followed by Tukey-Kramer and Dunnett multiple comparisons post tests, respectively. The mean correlation (Pearson's) coefficients for intrajudge and interjudge analyses of the densitometric quantitations were in the range of $0.89-0.96$.

\section{RESULTS}

\section{Specific knockdown of GLT-1 and EAAC1 proteins by antisense ODNs}

Compared with aCSF infusion, GLT-1 antisense (but not sense or random) infusion for $5 \mathrm{~d}$ significantly reduced GLT-1 immunoreactive protein levels in the cerebral cortex $(66 \pm 7 \%$; $p<$ $0.05 ; n=5$ per group) and striatum $(60 \pm 9 \% ; p<0.05 ; n=5$ per group) without affecting the levels of the cytoskeletal protein $\beta$-tubulin (Fig. 1). Hippocampus of the GLT-1 antisense-inf used rats also showed a $61 \pm 11 \%$ decrease in the GLT-1 protein levels. Previous studies showed that inf usion of GLT-1 antisense significantly reduces the GLT-1 protein levels in the striatum and hippocampus, but not in the cerebellum and spinal cord (Rothstein et al., 1996). Inf usion of EAAC1 antisense (but not sense or random) for $5 \mathrm{~d}$ specifically decreased EAAC1 protein levels in the cerebral cortex $(62 \pm 9 \% ; p<0.05 ; n=5$ per group) and striatum ( $67 \pm 13 \% ; p<0.05 ; n=5$ per group), compared with aCSF-inf used controls (Fig. 1).
Table 1. Effect of GLT-1 and EAAC1 knockdown on the infarct volume after transient MCAO

\begin{tabular}{lllll} 
ICV inf usion & $n$ & Cortex $\left(\mathrm{mm}^{3}\right)$ & Striatum $\left(\mathrm{mm}^{3}\right)$ & Total $\left(\mathrm{mm}^{3}\right)$ \\
\hline None & 6 & $172 \pm 27$ & $31 \pm 5$ & $203 \pm 28$ \\
aCSF & 5 & $175 \pm 36$ & $37 \pm 7$ & $208 \pm 39$ \\
GLT-1 sense & 5 & $167 \pm 34$ & $34 \pm 9$ & $204 \pm 41$ \\
GLT-1 random & 4 & $164 \pm 33$ & $32 \pm 6$ & $199 \pm 47$ \\
GLT-1 antisense & 8 & $246 \pm 50^{a, b}$ & $48 \pm 7^{a, b}$ & $294 \pm 52^{a, b}$ \\
EAAC1 sense & 4 & $186 \pm 44$ & $33 \pm 10$ & $222 \pm 47$ \\
EAAC1 random & 4 & $175 \pm 49$ & $36 \pm 9$ & $210 \pm 52$ \\
EAAC1 antisense & 6 & $201 \pm 46$ & $39 \pm 7$ & $240 \pm 50$
\end{tabular}

Values are mean $\pm \mathrm{SD}$. The MCAO duration was $1 \mathrm{hr}$ in all cases. Ischemic injury volumes were computed by volumetric analysis of the thionine-stained coronal sections using the NIH Image program. aCSF, Artificial CSF; ICV, intracerebroventricular.

${ }^{a} p<0.05$, compared with the None or aCSF group (one-way ANOVA followed by Dunnet's multiple comparisons post test).

${ }^{b} p<0.05$, compared with the respective sense or random group (ANOVA followed by Tukey-Kramer multiple comparisons post test).

\section{GLT-1 antisense knockdown increased the ischemic infarct volume}

In SHR rats, $1 \mathrm{hr}$ transient MCAO and $24 \mathrm{hr}$ reperfusion resulted in an infarct in cerebral cortex $\left(172 \pm 27 \mathrm{~mm}^{3}\right)$ and striatum $\left(31 \pm 5 \mathrm{~mm}^{3}\right)$ with a total volume of $203 \pm 28 \mathrm{~mm}^{3}$ (Table 1$)$. Infusion of aCSF or GLT-1 sense/random resulted in no significant change in the transient MCAO-induced infarct volume. Infusion of GLT-1 antisense led to a significant increase in the cortical (43 $\pm 7 \% ; p<0.05)$, striatal $(55 \pm 9 \% ; p<0.05)$, and total $(45 \pm 9 \% ; p<0.05)$ infarct volume after transient MCAO, compared with the sense/random-infused controls (Table 1). Infusion of EAAC1 sense/random or antisense had no significant effect on the infarct volume after transient MCAO (Table 1). Figures 2 and 3 show the thionine-stained, serial coronal sections from the brains of representative rats that underwent transient MCAO or sham operation after infusion of GLT-1 (Fig. 2) and EAAC1 (Fig. 3) antisense and sense. There were no statistically significant differences between the groups infused with aCSF, GLT-1, or EAAC1 antisense, sense, or random in any of the physiological parameters (mean arterial blood pressure, $\mathrm{pH}$, $\mathrm{Pa}_{\mathrm{CO}_{2}}, \mathrm{~Pa}_{\mathrm{O}_{2}}$, hemoglobin, blood glucose, temporalis muscle, and rectal temperatures) (Table 2) and $\mathrm{rCBF}$ monitored during MCAO with a laser Doppler (Fig. 4). The end-point rCBF measured at $1 \mathrm{hr}$ of MCAO, using $\left[{ }^{14} \mathrm{C}\right] \mathrm{AIP}$ autoradiography, was also not significantly different between the GLT-1 sense- and antisense-infused rats or between the EAAC1 sense- and antisense-infused rats (Fig. 5).

\section{GLT-1 knockdown exacerbated the ischemic neuronal damage}

Microscopic analysis of the thionine-stained brain sections showed no observable histopathological changes in the aCSF and GLT-1 or EAAC1 sense/random-infused rats subjected to sham operation. No observable differences were found between the rats that underwent MCAO without any infusion and those infused with aCSF, GLT-1, or EAAC1 sense/random. GLT-1 antisense-infused/sham rats showed mild neuronal damage in both cortex (Fig. 6, top, second panel) and striatum (Fig. 6, bottom, second panel), compared with the cortex (Fig. 6, top, first panel) and striatum (Fig. 6, bottom, first panel) of the GLT-1 sense-inf used/sham control. Transient MCAO/reperfusion ( $1 \mathrm{hr} / 24 \mathrm{hr})$ in GLT-1 sense-infused rats resulted in a moderate neuronal damage in the cortex, with a 


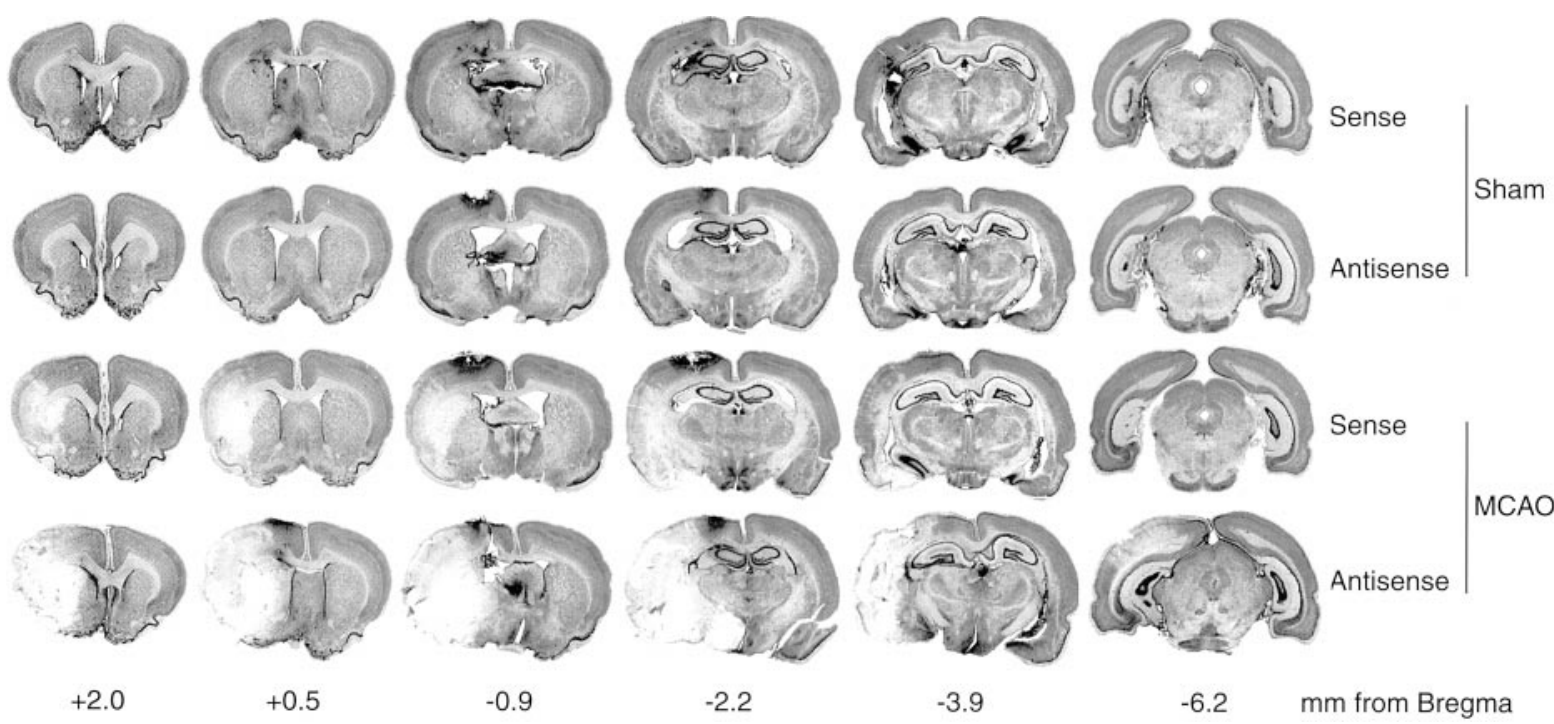

Figure 2. Thionine-stained serial coronal sections from the brains of GLT-1 antisense and sense ODN-infused rats that underwent transient MCAO $(1 \mathrm{hr})$ or sham operation. This figure shows only the GLT-1 sense-inf used control, because there were no observable differences between the GLT-1 sense- and random-infused groups. Transient MCAO in GLT-1 antisense-inf used rats resulted in significantly bigger infarcts compared with GLT-1 sense-inf used controls.

noticeable loss of layer $\mathrm{V}$ pyramidal neurons, loosening of neuropil in layers $\mathrm{V}$ and VI, and increased glial reactivity in the whole cortical infarct (Fig. 6, top, third panel). In the striatum of the GLT-1 sense-infused/MCAO group, the medium-sized neurons showed ischemic morphology (shrunken, pink, and eosinophilic), whereas the large striatal neurons were normal (Fig. 6, bottom, third panel ). In the GLT-1 antisense-inf used/MCAO group, there was a severe neuronal loss in all six cortical layers, with a nearly total loss of the large pyramidal neurons from layer $\mathrm{V}$ and evident glial infiltration in the cortex (Fig. 6, top, fourth panel). The striatum of the GLT-1 antisense-infused/MCAO group also showed severe loss of the medium-sized neurons (Fig. 6, bottom, fourth panel), and the surviving neurons were either shrunken and eosinophilic or chromolytic and hyperchromatic, surrounded by astrocytes and microglia.

\section{Transient MCAO after GLT-1 knockdown increased the neurological deficit and mortality}

Transient MCAO ( $1 \mathrm{hr}$ ) resulted in a mild to moderate neurological deficit at $24 \mathrm{hr}$ of reperfusion, with a mortality rate of $\sim 14 \%$ during the first $24 \mathrm{hr}$ of reperfusion (Table 3 ). Inf usion of aCSF, GLT-1 sense or random had no significant effect on the transient MCAO-induced neurological deficit or mortality rate (Table 3), whereas transient MCAO in the GLT-1 antisense-inf used rats resulted in severe to very severe neurological deficit with an $\sim 33 \%$ mortality rate (Table 3 ). EAAC1 antisense infusion had no significant effect on the transient MCAO-induced neurological deficit or mortality rate (Table 3). Sham rats inf used with aCSF, GLT-1, or EAAC1 antisense/ sense/random showed no neurological deficit or significant mortality (Table 3).

\section{DISCUSSION}

The results of this study show that GLT-1 knockdown increases the susceptibility of rats to infarct development and neuronal death after transient MCAO. Transient MCAO after GLT-1 knockdown also worsened the neurological status and increased the mortality rate; however, the increased size of the infarct may be responsible for these factors. EAAC1 knockdown had no effect
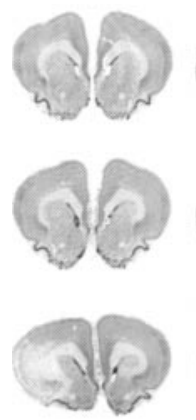

Figure 3. Thionine-stained serial coronal sections from the brains of EAAC1 antisense and sense ODN-infused rats that underwent transient MCAO (1 hr) or sham operation. EAAC1 knockdown had no significant effect on the infarct size.

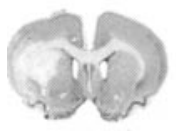

$+2.0$
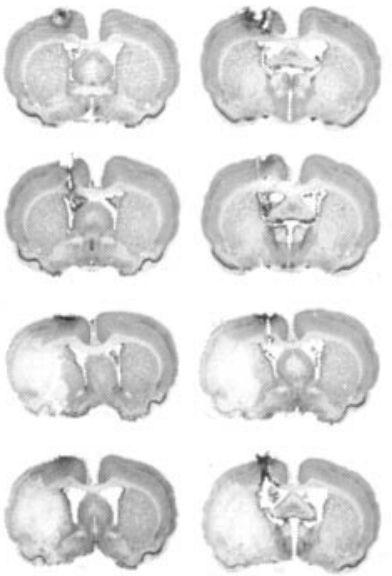

$+0.5$

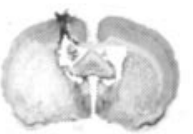

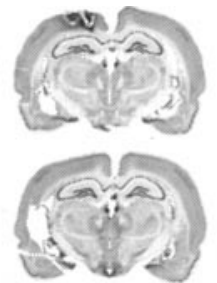

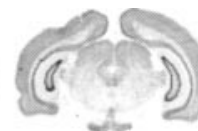

Sense

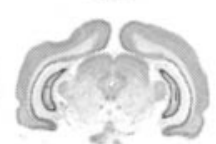

Antisense

Sham

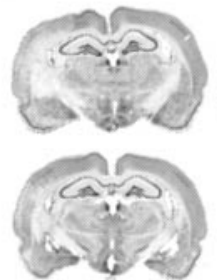

$-3.9$

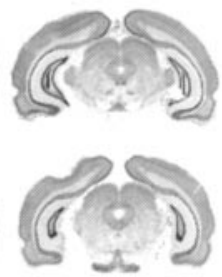

Sense

$-0.9$ 
Table 2. Physiological parameters in GLT-1 and EAAC1 sense, random, and antisense ODN-infused rats undergoing transient MCAO

Temperature $\left({ }^{\circ} \mathrm{C}\right)$

\begin{tabular}{|c|c|c|c|c|c|c|c|c|}
\hline ICV infusion & $n$ & $\mathrm{pH}$ & $\mathrm{PaCO}_{2}(\mathrm{~mm} \mathrm{Hg})$ & $\mathrm{PaO}_{2}(\mathrm{~mm} \mathrm{Hg})$ & Gl (mg/dl) & $\mathrm{Hb}(\mathrm{gm} / \mathrm{dl})$ & Rectal & Muscle \\
\hline None & 6 & $7.41 \pm 0.04$ & $34.2 \pm 3.3$ & $136 \pm 22$ & $90 \pm 6$ & $13 \pm 1.3$ & $36.9 \pm 0.4$ & $36.5 \pm 0.4$ \\
\hline $\mathrm{aCSF}$ & 5 & $7.38 \pm 0.03$ & $32.4 \pm 2.6$ & $133 \pm 30$ & $91 \pm 5$ & $14 \pm 1.6$ & $37.2 \pm 0.4$ & $36.3 \pm 0.5$ \\
\hline GLT-1 sense & 5 & $7.42 \pm 0.05$ & $33.7 \pm 3.6$ & $142 \pm 34$ & $91 \pm 8$ & $13 \pm 2.1$ & $37.1 \pm 0.5$ & $36.5 \pm 0.4$ \\
\hline GLT-1 random & 4 & $7.40 \pm 0.04$ & $33.3 \pm 5.1$ & $132 \pm 31$ & $88 \pm 7$ & $14 \pm 1.7$ & $37.4 \pm 0.6$ & $36.4 \pm 0.3$ \\
\hline GLT-1 antisense & 8 & $7.38 \pm 0.05$ & $31.6 \pm 4.8$ & $138 \pm 37$ & $92 \pm 8$ & $14 \pm 1.5$ & $37.0 \pm 0.5$ & $36.7 \pm 0.4$ \\
\hline EAAC1 sense & 4 & $7.39 \pm 0.03$ & $32.2 \pm 2.1$ & $133 \pm 25$ & $92 \pm 8$ & $13 \pm 1.2$ & $36.9 \pm 0.6$ & $36.7 \pm 0.3$ \\
\hline EAAC1 random & 4 & $7.42 \pm 0.04$ & $34.8 \pm 4.5$ & $140 \pm 38$ & $93 \pm 5$ & $14 \pm 1.3$ & $37.2 \pm 0.6$ & $36.8 \pm 0.2$ \\
\hline EAAC1 antisense & 6 & $7.43 \pm 0.05$ & $32.9 \pm 3.8$ & $137 \pm 29$ & $89 \pm 6$ & $13 \pm 1.9$ & $37.3 \pm 0.5$ & $36.5 \pm 0.5$ \\
\hline
\end{tabular}

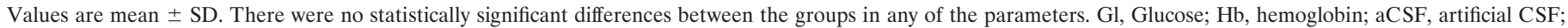

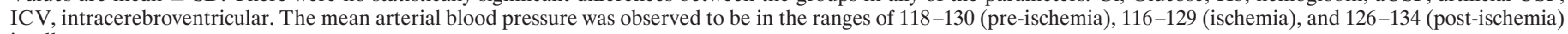
in all groups.

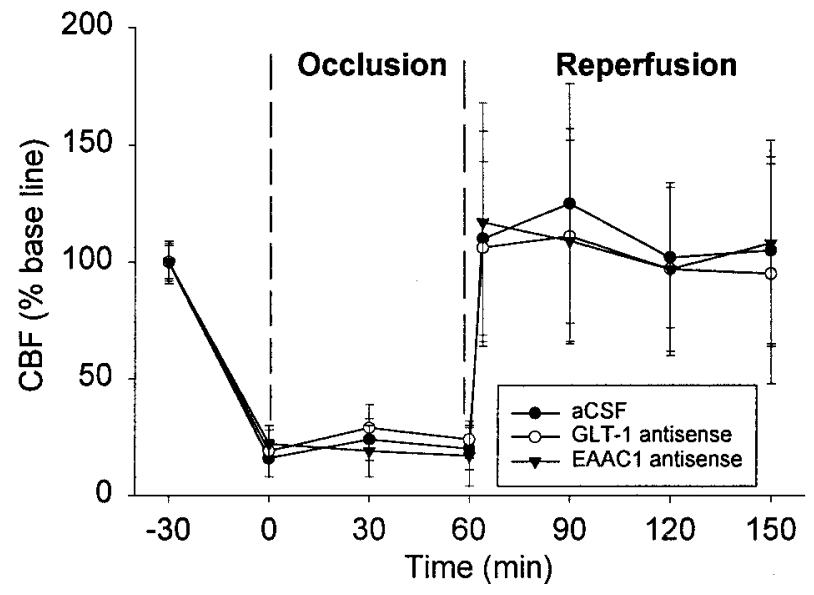

Figure 4. Effect of GLT-1 and EAAC1 antisense ODN infusion on $\mathrm{rCBF}$ after transient MCAO. The rCBF was measured using a laser Doppler flowmeter probe placed on the surface of the ipsilateral cortex (ischemic area) through a craniectomy over the MCA territory. Changes are expressed as percentage of the baseline. Values are mean \pm SD. There were no statistically significant differences between the groups. Infusion of GLT-1 and EAAC1 sense and random led to no significant alterations in the $\mathrm{rCBF}$ after MCAO (data not shown).

on the transient MCAO-induced neuronal death. This is the first study showing that GLT-1 dysf unction exacerbates ischemic neuronal damage.

Although there are at least five subtypes of glutamate transporters in mammalian CNS (Kanai et al., 1997; Seal and Amara, 1999), GLT-1 is the predominant subtype that carries the bulk of glutamate reuptake (Robinson, 1999). Antisense knockdown of GLT-1 protein in normoxic rat brain was shown to induce increased extracellular glutamate levels and neuronal damage (Rothstein et al., 1996). Although GLT-1-deficient transgenic mice showed selective neuronal damage in the hippocampal CA1 region and increased susceptibility to edema formation after cold-induced cortical injury (Tanaka et al., 1997), GLASTdeficient mice showed no apparent neuronal damage in any brain region but developed more edema in cerebellum after coldinduced cerebellar injury (Watase et al., 1998). These studies show that proper functioning of both of the glial glutamate transporters is important for preventing excitotoxic neuronal damage, but their role depends on their known structural localization within the brain. GLT-1 and GLAST were reported to be more abundant in cerebral cortex and cerebellum, respectively
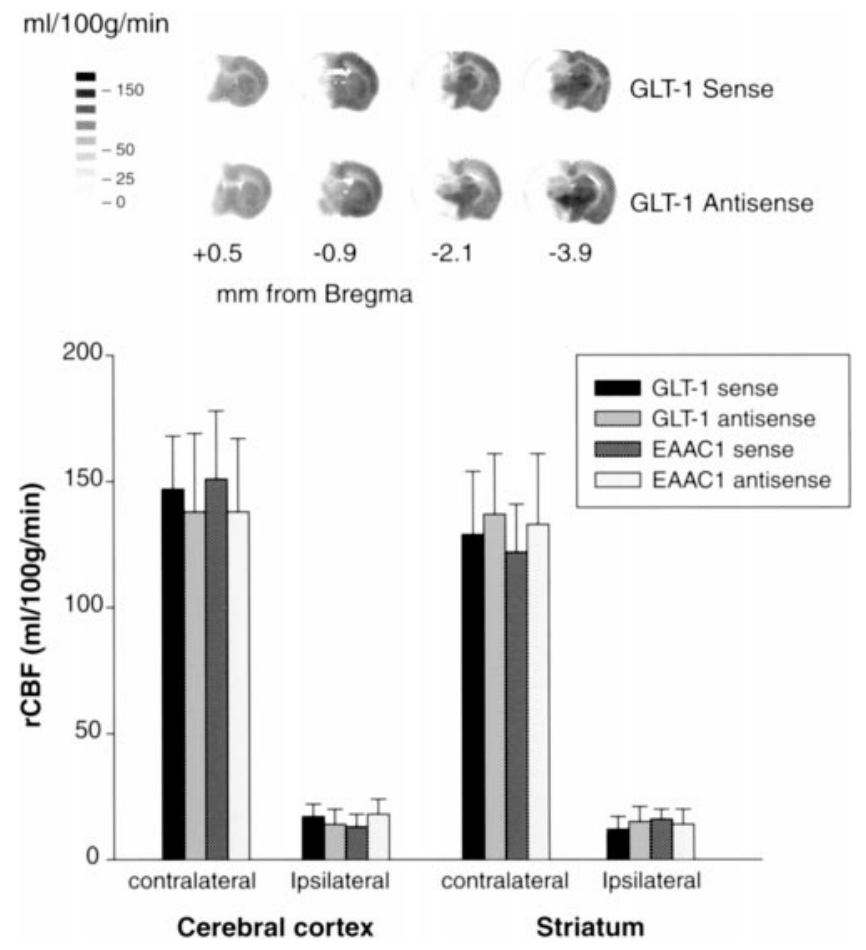

Figure 5. End-point rCBF rates at $1 \mathrm{hr}$ of $\mathrm{MCAO}$ as measured by autoradiography using $\left[{ }^{14} \mathrm{C}\right]$ AIP in the GLT- 1 sense $(n=5)$, GLT- 1 antisense $(n=5)$, EAAC1 sense $(n=4)$, and EAAC1 antisense $(n=$ 4)-infused rats. Flow rates in the representative areas of cerebral cortex and striatum were averaged across the contralateral and ipsilateral sides of the brain. No significant differences were observed between GLT-1 senseand antisense-infused groups and EAAC1 sense- and antisense-infused groups. The inset shows autoradiographs generated using the coronal brain sections $(+0.5,-0.9,-2.1$, and $-3.9 \mathrm{~mm}$ from bregma) of $\left[{ }^{14} \mathrm{C}\right] \mathrm{AIP}$-administered GLT-1 sense- and antisense-infused groups.

(Storck et al., 1992; Torp et al., 1994). The present antisense studies demonstrated that optimal functioning of GLT-1 in cerebrum is essential for minimizing the neuronal damage after stroke.

Extracellular glutamate levels increase after transient MCAO (Takagi et al., 1994; Uchiyama-Tsuyuki et al., 1994), which may lead to excitotoxic neuronal death by overstimulating the NMDA receptors. Previous studies showed that treatment with NMDA receptor ion channel blockers, glycine site antagonists, competitive and noncompetitive NMDA binding site antagonists, and 
Figure 6. Microscopic evaluation of the cerebral cortex (top panels) and striatum (bottom panels) from GLT-1 sense and antisense ODN-infused rats subjected to either sham operation or transient MCAO. The cortical neuronal layers (top panels) are indicated by $I-V I$. Severe neuronal loss in all cortical layers with a nearly total loss of the large pyramidal neurons from layer $\mathrm{V}$ and evident glial infiltration can be seen in the brains of GLT-1 antisense-inf used/MCAO group. The striatum (bottom panels) of the GLT-1 antisense-infused/MCAO group also showed severe loss of the mediumsized striatal neurons (arrowheads), whereas the large striatal neurons (arrows) survived. The Figure shows only the sense-infused control, because there was no observable difference between sense- and random-inf used groups.
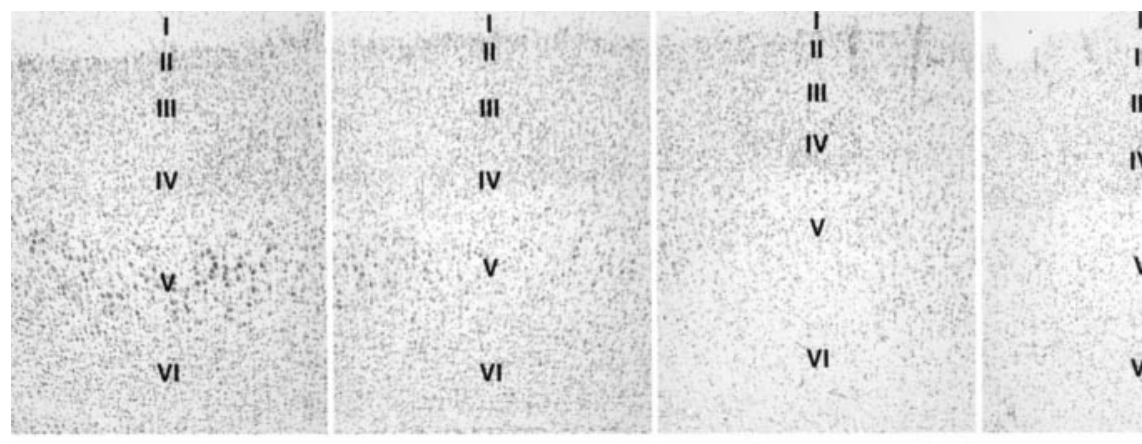

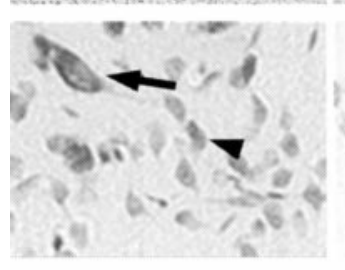

Sense/Sham

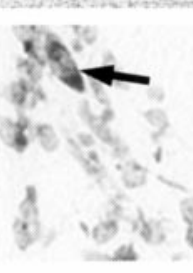

Antisense/Sham

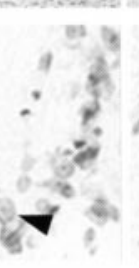

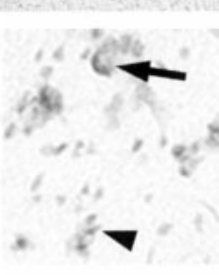

Sense/Ischemia
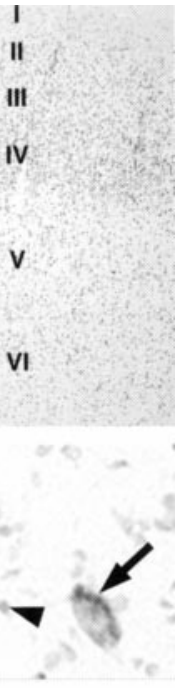

Antisense/Ischemia

Table 3. Mortality rates and neuroscores after sham operation and transient MCAO in GLT-1 and EAAC1 sense, random, and antisense ODNinfused rats

\begin{tabular}{|c|c|c|c|c|c|}
\hline \multirow[b]{2}{*}{ ICV infusion } & \multirow{2}{*}{$\begin{array}{l}\text { Total rats } \\
\text { (alive/dead) }\end{array}$} & \multirow[b]{2}{*}{$\%$ mortality } & \multicolumn{2}{|l|}{ Neuroscores } & \multirow[b]{2}{*}{ Neurological deficit } \\
\hline & & & Individual & Median & \\
\hline \multicolumn{6}{|l|}{ Sham-operated } \\
\hline None & $6(6 / 0)$ & 0 & $0,0,0,0,0,0$ & 0 & None \\
\hline $\mathrm{aCSF}$ & $5(5 / 0)$ & 0 & $0,0,0,0,0$ & 0 & None \\
\hline GLT-1 sense & $5(5 / 0)$ & 0 & $0,0,0,1,1$ & 0.40 & None \\
\hline GLT-1 random & $4(4 / 0)$ & 0 & $0,0,0,1$ & 0.25 & None \\
\hline GLT-1 antisense & $8(7 / 1)$ & 12 & $0,0,0,1,1,1,1$ & 0.57 & None \\
\hline EAAC1 sense & $4(4 / 0)$ & 0 & $0,0,0,0$ & 0 & None \\
\hline EAAC1 random & $4(4 / 0)$ & 0 & $0,0,0,0$ & 0 & None \\
\hline EAAC1 antisense & $6(5 / 1)$ & 16 & $0,0,0,0,1$ & 0.20 & None \\
\hline \multicolumn{6}{|l|}{ Transient MCAO } \\
\hline None & $7(6 / 1)$ & 14 & $1,2,2,2,2,2$ & $1.83^{a}$ & Mild-moderate \\
\hline $\mathrm{aCSF}$ & $6(5 / 1)$ & 16 & $1,2,2,2,2$ & $1.80^{a}$ & Mild-moderate \\
\hline GLT-1 sense & $5(5 / 0)$ & 0 & $1,2,2,2,3$ & $2.00^{a}$ & Mild-moderate \\
\hline GLT-1 random & $5(4 / 1)$ & 20 & $1,2,2,2$ & $1.75^{a}$ & Mild-moderate \\
\hline GLT-1 antisense & $12(8 / 4)$ & $33^{a, b}$ & $3,3,3,3,3,4,4,4$ & $3.38^{a, b}$ & Severe-very severe \\
\hline EAAC1 sense & $4(4 / 0)$ & 0 & $1,2,2,2$ & $1.75^{a}$ & Mild-moderate \\
\hline EAAC1 random & $4(4 / 0)$ & 0 & $1,2,2,2$ & $1.75^{a}$ & Mild-moderate \\
\hline EAAC1 antisense & $7(6 / 1)$ & 14 & $1,1,2,2,2,3$ & $1.83^{a}$ & Mild-moderate \\
\hline
\end{tabular}

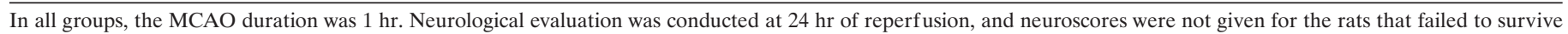

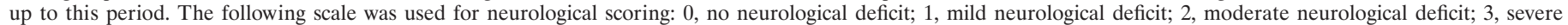
neurological deficit; and 4, very severe neurological deficit. ICV, Intracerebroventricular; aCSF, artificial CSF.

${ }^{a} p<0.05$, compared with the corresponding sham control (one-way ANOVA followed by Tukey-Kramer multiple comparisons post test).

${ }^{b} p<0.05$, compared with the respective sense/MCAO or random/MCAO control (one-way ANOVA followed by Tukey-Kramer multiple comparisons post test).

antisense ODN against NMDA receptor NR1 subunit reduce the infarct volume, or the area of significant cell necrosis, caused by permanent or transient focal ischemia (for review, see Lipton, 1999). A slower rate of clearance of the released glutamate may be the major consequence of GLT-1 knockdown. Increased release (caused by ischemia) and decreased reuptake (caused by knockdown) together may lead to an increase in the duration of glutamate action in the synaptic cleft and may have caused more NMDA receptor stimulation and exacerbated ischemic neuronal damage after GLT-1 knockdown. Previous studies showed that dysfunctional glutamate transport and cerebral energy failure synergistically promote excitotoxic neuronal death (Massieu and
Tapia, 1997; Massieu and Gomez-Raman, 1999; SanchezCarbente and Massieu, 1999). Thus, cerebral energy failure during the ischemic period may also be responsible for the exacerbated neuronal damage after GLT-1 knockdown.

On the basis of observations that the nonspecific sodiumdependent glutamate uptake blocker DL-TBOA reduces the forebrain ischemia-induced glutamate release by the same magnitude as DHK, Phillis et al. (2000) suggested that GLT-1 reversal may be an important event in ischemic glutamate release. A recent preliminary study showed that GLT-1 exists as two subspecies, viz GLT-1a (glial) and GLT-1b (glial and neuronal), and the two forms are indistinguishable with respect to their $K_{\mathrm{m}}$ values, as 
well as to the $K_{\mathrm{i}}$ values of L-trans-pyrrolidine-2,4-dicarboxylate, DHK, and serine- $O$-sulfate (Chen et al., 2000). Seki et al. (1999) showed that infusion of GLT-1-preferring inhibitor DHK significantly attenuated the forebrain ischemia-induced glutamate release from striatum and suggested GLT-1 reversal as a cause of ischemia-induced glutamate release. It is unclear from this study whether forebrain ischemia caused neuronal damage in striatum and the inhibition of glutamate release by DHK led to neuroprotection. However, infusion of DHK induces neuronal damage in the striatum and hippocampus of normal rats (Massieu and Tapia, 1997). Longuemare and Swanson (1995) showed that cultured astrocytes subjected to energy failure release glutamate via the reversal of sodium-dependent uptake. However, Ottersen et al. (1996) observed increased astrocytic glutamate content after ischemia. Recent studies also showed that DHK had no effect on glutamate release and anoxic depolarization current in the hippocampal slices subjected to in vitro ischemia (Roettger and Lipton, 1996; Rossi et al., 2000). These two studies indicate against GLT-1 reversal as a cause of ischemic glutamate release. The present antisense studies show increased ischemic neuronal damage after GLT-1 knockdown, supporting the concept that GLT-1 reversal may not be responsible for glutamate release during in vivo ischemia.

Because EAAC1 was reported to be absent in the glutamatergic nerve terminals (Danbolt et al., 1998), its reversal may not be responsible for ischemia-induced extracellular glutamate concentrations. If EAAC1 reversal is responsible for glutamate release, induction of ischemia after EAAC1 knockdown should be neuroprotective. However, this study failed to observe either decrease or exacerbation of ischemic neuronal damage after EAAC1 knockdown, suggesting against EAAC1 as a contributor of glutamate release after ischemia. Because the antisense knockdown decreases EAAC1 protein by only $\sim 65 \%$, induction of ischemia in EAAC1 gene knockout mice may clarify beyond a doubt the role of EAAC1 in glutamate release and the neuronal damage beyond doubt.

Because glutamate transporters are dynamic proteins that could release as well as reuptake glutamate, their function depends on their regional and cellular localization, cerebral energy status, and the duration and type of the neuronal insult. Furthermore, most of the studies proposing transporter reversal responsible for glutamate release in ischemia were conducted with in vitro ischemia paradigms (using either the cultured cells or the hippocampal slices) that are very different from the in vivo ischemia studies. An in vivo study using a rat forebrain ischemia model showed that glutamate transporter inhibitor threo-3hydroxy-DL-aspartate (TBHA) does not block the ischemiainduced glutamate release (Heron et al., 1995). However, this study had not demonstrated whether TBHA competes with glutamate for the transporter sites from the inner side of the synaptic membrane. Results of the present study suggest that whatever may be the mechanism of glutamate release during ischemia, knockdown of GLT-1 (but not EAAC1) exacerbates the transient MCAO-induced neuronal damage.

GLT-1 stimulators may be useful in preventing ischemic neuronal damage. Although recent studies showed that the glialderived neurotrophic factor and pituitary adenylate cyclaseactivating polypeptide induce GLT-1 expression (Coccia et al., 1999; Figiel and Engele, 2000), additional studies are needed to prove their usefulness in neuropathological conditions associated with GLT-1 dysfunction. The neuroprotective potential of the neural stem cells transfected with GLT-1 to prevent excitotoxicity is under investigation and may prove to be useful in the future (Poulsen et al., 2000).

\section{REFERENCES}

Alkayed NJ, Harukuni I, Kimes AS, London ED, Traystman RJ, Hurn PD (1998) Gender-linked brain injury in experimental stroke. Stroke 29:159-166.

Chaudhry FA, Lehre KP, Campangne ML, Otterson OP, Danbolt NC, Storm-Mathisen J (1995) Glutamate transporters in glial plasma lemma membranes: highly differentiated localizations revealed by quantitative ultrastructural immunocytochemistry. Neuron 15:717-720.

Chen W, Aoki C, Gruber C, Hadley R, Wang G, Blitzblau R, Volpe JJ, Irwin N, Rosenberg PA (2000) Molecular cloning, functional characterization, and neuronal localization of a variant form of the glutamate transporter GLT-1. Soc Neurosci Abstr 26:539.15.

Coccia C, Ganel R, Rothstein JD (1999) GDNF induces an increase in EAAT2 (GLT-1) expression. Soc Neurosci Abstr 25:170.2.

Danbolt NC, Chaudhry FA, Dehnes Y, Lehre KP, Levy LM, Ullensvang K, Storm-Mathisen J (1998) Properties and localization of glutamate transporters. Prog Brain Res 116:23-43.

Dogan A, Rao AM, Hatcher J, Rao VLR, Baskaya MK, Dempsey RJ (1999) Effects of MDL 72527, a specific inhibitor of polyamine oxidase, on brain edema, ischemic injury volume, and tissue polyamine levels in rats after temporary middle cerebral artery occlusion. J Neurochem 72:765-770.

Figiel M, Engele J (2000) Pituitary adenylate cyclase-activating polypeptide (PACAP), a neuron-derived peptide regulating glial glutamate transport and metabolism. J Neurosci 20:3596-3605.

Heron A, Springhetti V, Seylaz J, Lasbennes F (1995) Effects of a glutamate uptake inhibitor on glutamate release induced by veratridine and ischemia. Neurochem Int 26:593-599.

Inage YW, Itoh M, Wada K, Takashima S (1998) Expression of two glutamate transporters, GLAST and EAAT4, in the human cerebellum: their correlation in development and neonatal hypoxic-ischemic damage. J Neuropathol Exp Neurol 57:554-562.

Kanai Y, Hediger MA (1992) Primary structure and functional characterization of a high-affinity glutamate transporter. Nature 360:467-471.

Kanai Y, Trotti D, Nussberger S, Hediger MA (1997) The high-affinity glutamate transporter family. In: Neurotransmitter transporters: structure, function, and regulation (Reith MEA, ed), pp 171-213. Totowa, NJ: Humana.

Kato H, Kogure K (1999) Biochemical and molecular characteristics of the brain with developing cerebral infarction. Cell Mol Neurobiol 19:93-108.

Lipton P (1999) Ischemic cell death in brain neurons. Physiol Rev 79:1431-1568.

Longa EZ, Weinstein PR, Carlson S, Cummins R (1989) Reversible middle cerebral artery occlusion without craniectomy in rat. Stroke 20:84-91

Longuemare MC, Swanson RA (1995) Excitatory amino acid release from astrocytes during energy failure by reversal of sodium-dependent uptake. J Neurosci Res 40:379-386.

Martin LJ, Brambrink AM, Lehmann C, Portera-Cailliau C, Koehler R, Rothstein J, Traystman RJ (1997) Hypoxia-ischemia causes abnormalities in glutamate transporters and death of astroglia and neurons in newborn pig striatum. Ann Neurol 42:335-348.

Massieu L, Gomez-Raman N (1999) Glycolysis inhibition exacerbates neuronal damage induced by glutamate transporter inhibition in vivo. J Neurochem [Suppl] 73:S96D.

Massieu L, Tapia R (1997) Glutamate uptake impairment and neuronal damage in young and aged rats in vivo. J Neurochem 69:1151-1160.

Ottersen OP, Laake JH, Reichelt W, Haug FM, Torp R (1996) Ischemic disruption of glutamate homeostasis in brain: quantitative immunocytochemical analyses. J Chem Neuroanat 12:1-14.

Paxinos G, Watson C (1998) The rat brain in stereotaxic coordinates, Ed 4. New York: Academic.

Phillis JW, O'Regan MH (1996) Mechanisms of glutamate and aspartate release in the ischemic rat cerebral cortex. Brain Res 730:150-164.

Phillis JW, Ren J, O'Regan MH (2000) Transporter reversal as a mechanism of glutamate release from the ischemic rat cerebral cortex: studies with DL-threo- $\beta$-benzyloxyaspartate. Brain Res 868:105-112.

Pines G, Danbolt NC, Bjoras M, Zhang Y, Bendahan A, Eide L, Koepsell H, Storm-Mathisen J, Seeberg E, Kanner BI (1992) Cloning and expression of a rat brain L-glutamate transporter. Nature 360:464-467.

Poulsen D, Maragakis NJ, Hoke A, Snyder EY, During MJ, Rothstein JD (2000) EAAT2 transfected murine neural stem cells demonstrate glutamate uptake and are capable of engraftment in organotypic spinal cord cultures. Soc Neurosci Abstr 26:327.11.

Rao VLR, Baskaya MK, Dogan A, Rothstein JD, Dempsey RJ (1998) Traumatic brain injury down-regulates glial glutamate transporter (GLT-1 and GLAST) proteins in rat brain. J Neurochem 70:2020-2027.

Rao VLR, Rao AM, Dogan A, Bowen KK, Hatcher J, Rothstein JD, 
Dempsey RJ (2000) Glial glutamate transporter GLT-1 downregulation precedes delayed neuronal death in gerbil hippocampus following transient global cerebral ischemia. Neurochem Int 36:531-537.

Robinson MB (1999) The family of sodium-dependent glutamate transporters: a focus on the GLT-1/EAAT2 subtypes. Neurochem Int 33:479-491.

Roettger V, Lipton P (1996) Mechanism of glutamate release from rat hippocampal slices during in vitro ischemia. Neuroscience 75:677-685.

Rossi DJ, Oshima T, Attwell D (2000) Glutamate release in severe brain ischemia is mainly by reversed uptake. Nature 403:316-321.

Rothstein JD, Martin LJ, Levey AI, Dykes-Hoberg M, Jin L, Wu D, Nash N, Kuncl RW (1994) Localization of neuronal and glial glutamate transporters. Neuron 13:713-725.

Rothstein JD, Dykes-Hoberg M, Pardo CA, Bristol LA, Jin L, Kuncl RW, Kanai Y, Hediger MA, Wang Y, Schielke JP, Welty DF (1996) Knockout of glutamate transporters reveals a major role for astroglial transport in excitotoxicity and clearance of glutamate. Neuron 16:675-686.

Rusa R, Alkayed NJ, Crain BJ, Traystman RJ, Kimes AS, London ED, Klaus JA, Hurn PD (1999) $17 \beta$-Estradiol reduces stroke injury in estrogen-deficient female animals. Stroke 30:1665-1670.

Sanchez-Carbente MR, Massieu L (1999) Transient inhibition of glutamate uptake in vivo induces neurodegeneration when energy metabolism is impaired. J Neurochem 72:129-138.

Seal RP, Amara SG (1999) Excitatory amino acid transporters: a family in flux. Annu Rev Pharmacol Toxicol 39:431-456.

Seki Y, Feustel PJ, Keller RW, Tranmer BI, Kimelberg HK (1999) Inhibition of ischemia-induced glutamate release in rat striatum by dihydrokinate and an anion channel blocker. Stroke 30:433-440.

Storck T, Schulte S, Hoffman K, Stoffel W (1992) Structure, expression, and functional analysis of a $\mathrm{Na}^{+}$-dependent glutamate/aspartate transporter from rat brain. Proc Natl Acad Sci USA 89:10955-10959.
Swanson RA, Morton MT, Tsao-Wu G, Savalos RA, Davidson C, Sharp FR (1990) A semiautomated method for measuring brain infarct volume. J Cereb Blood Flow Metab 10:290-293.

Takagi K, Ginsberg MD, Globus MY, Martinez E, Busto R (1994) Effect of hyperthermia on glutamate release in ischemic penumbra after middle cerebral artery occlusion in rats. Am J Physiol 267:H1770-1776.

Tanaka K, Watase K, Manabe T, Yamada K, Watanabe M, Takahashi K, Iwama H, Nishikawa T, Ichihara N, Kikuchi T, Okuyama S, Kawashima N, Hori S, Takimoto M, Wada K (1997) Epilepsy and exacerbation of brain injury in mice lacking the glutamate transporter GLT-1. Science 276:1699-1702.

Torp R, Danbolt NC, Babaie E, Bjoras M, Seeberg E, Storm-Mathisen J, Ottersen OP (1994) Differential expression of two glial glutamate transporters in the rat brain: an in situ hybridization study. Eur J Neurosci 6:936-942.

Torp R, Lekieffre D, Levy LM, Haug FM, Danbolt NC, Meldrum BS, Ottersen OP (1995) Reduced postischemic expression of a glial glutamate transporter, GLT1, in the rat hippocampus. Exp Brain Res 103:51-58.

Uchiyama-Tsuyuki Y, Araki H, Yae T, Otomo S (1994) Changes in the extracellular concentrations of amino acids in the rat striatum during transient focal cerebral ischemia. J Neurochem 62:1074-1078.

Watase K, Hashimoto K, Kano M, Yamada K, Watanabe M, Inoue Y, Okuyama S, Sakagawa T, Ogawa S, Kawashima N, Hori S, Takimoto M, Wada K, Tanaka K (1998) Motor discoordination and increased susceptibility to cerebellar injury in GLAST mutant mice. Eur J Neurosci 10:976-988.

Zhang RL, Chopp M, Chen H, Garcia JH (1994) Temporal profile of ischemic tissue damage, neutrophil response, and vascular plugging following permanent and transient $(2 \mathrm{H})$ middle cerebral artery occlusion in the rat. J Neurol Sci 125:3-10. 